\title{
Bioactive Compounds: The Key to Functional Foods
}

\section{Danik Martirosyan ${ }^{1}$ and Emma Miller ${ }^{2}$}

${ }^{1}$ Functional Food institute, Dallas, TX, USA; ${ }^{2}$ Cornell University, Ithaca, NY, USA

Submission Date: July $27^{\text {th }}, 2018$, Acceptance Date: July $30^{\text {th }}, 2018$, Publication Date: July $31^{\text {st }}$, 2018

Citation: Martirosyan D., Miller E., Bioactive Compounds: The Key to Functional Foods. Bioactive Compounds in Health and Disease 2018; 1(3): 36-39. DOI: https://doi.org/10.31989/bchd.v1i3.539

\section{EDITORIAL}

Bioactive compounds are the constituents of foods, especially functional foods, that provide beneficial health properties. For example, these benefits include antioxidants, anti-inflammatory, antifungal, and various additional preventative properties, which illustrates how bioactive compounds are the real-life example of Hippocrates' notion "let thy food be thy medicine" [1]. Originally, many definitions of functional foods neglected to acknowledge the importance of bioactive compounds [2]. Bioactive compounds are the constituents that enhance functional foods and therefore are necessary to incorporate under the umbrella of functional foods. Functional foods are able to provide health benefits though bioactive compounds, as these compounds target mechanisms that manage, prevent, and/or treat disease [2]. The Functional Food Center asserts that bioactive compounds are quintessential of functional foods and essential for optimal health, thereby leading to the inclusion of bioactive compounds in the most recent definition of functional foods [1].

In order to appreciate the importance of bioactive compounds within the role of functional foods, bioactive compounds must be defined. Bioactive compounds are chemical components that contribute to regulating biological mechanisms. Through excessive research, bioactive compounds are more accurately defined by Dr. Martirosyan and Pisarski as the following: "food bioactive compounds are primary and secondary metabolites of nutritive and non-nutritive natural components generating health benefits by preventing or managing chronic disease or its symptoms" [1]. Since these compounds are antioxidants, anti-inflammatory, etc., bioactive compounds provide benefits to health [1]. Although they are a small proportion of a food source, these compounds can trigger mechanisms that will alter and improve human health. However, while bioactive compounds are extremely beneficial they can be consumed in excess, leading to toxicity. Therefore, each bioactive compound must be evaluated to establish a beneficial amount and a toxic threshold [1]. Toxicity and beneficial levels must be determined and established through clinical research. Diets containing the appropriate amount of bioactive compounds can be instrumental in functional foods providing the best function. 
The definition of functional foods is not regulated. Accordingly, many institutions have individual definitions. There is no one definition of functional foods accepted by all. Functional foods are generally defined as those that improve health and can help treat disease. The Functional Food Center previously defined functional foods as "Natural or processed foods that contain known or unknown biologically-active compounds; which, in defined, effective, and non-toxic amounts, provide a clinically proven and documented health benefit for the prevention, management, or treatment of chronic diseases" [2]. However, in 2017, the Functional Food Center updated their definition to: "natural or processed foods that contain biologically active compounds which, in defined, effect, and non-toxic amounts provide clinically proven and documented health benefit utilizing specific biomarker for the prevent, management, or treatment of chronic disease or its symptoms" [2]. The new change removes the notion of the unknown compounds, which acknowledges that there are bioactive compounds. Additionally, there is acknowledgement that bioactive compounds can also be instrumental in treating symptoms, not just the management of chronic disease. Countless studies on functional foods show "the intake of selected foods and their associated constituents can have profound physiologic effects." These effects are driven by the bioactive compounds in the functional foods [3]. Bioactive compounds have specific health benefits that contribute to the function of functional foods and should therefore be included the definition of functional foods.

Many institutions have their own definition of functional foods, as there is no regulated standard definition. For example, the Institute of Food Technologists define these foods as "foods and food components that provide a health benefit beyond basic nutrition. These substances provide essential nutrients often beyond quantities necessary for normal maintenance, growth, and development, and/or other biologically active components that impact health benefits" [5]. While this definition does mention biologically active components, there is a failure to discuss the important aspects of bioactive compounds that further ensure health. More specifically, there is no discussion of toxicity of an excess of bioactive compounds or a minimum level in the diet to obtain the benefits. Without the proper inclusion of bioactive compounds, the definition does not identify the important aspect of functional foods that interact with the biomarkers and improve health. The advancement in the Functional Food Center's definition generates a clearer notion of the mechanism of functional foods by identifying that bioactive compounds work on biomarkers, which indicate effectiveness [1].

A functional food, previously defined, is a food containing biologically active compounds that can be used to treat a disease and/or its symptoms. With the rise of chronic disease, effective treatment is in high demand [2]. The concept was originally introduced by the Japanese, with the idea that certain foods can work beyond mundane nutritional effects [6]. Functional foods target specific mechanisms that are linked to chronic diseases such as cancer and Alzheimer's disease [3]. Through targeting these mechanisms, functional foods accomplish what medication often fails to do: treat chronic disease. Functional foods, if they are consumed under the toxic dosage, effectively manage chronic diseases and their accompanied symptoms, without the excessive side effects that are associated with medication. However, medication frequently brings adverse side effects while functional foods, using bioactive compounds, can treat disease. [2]. Type 2 Diabetes 
is a chronic disease that falls within the conflict of which is the best treatment: medication or diet. Medication often works to help the person lose weight and regain insulin sensitivity, which is similar to what a diet in functional foods that target these mechanisms can do [7]. Functional foods further solidify Hippocrates's ideals, supporting the important idea to "let thy food be thy medicine", which suggests that functional food could also be medicine [1].

Type 2 Diabetes is not the only chronic disease that has been treated with functional foods. Various studies have explored the use of functional foods to treat cardiovascular diseases. For example, seaweeds, a functional food candidate, are used as a preventative measure when treating those at risk for heart disease [8]. The metabolites in seaweed, such as lipids and fiber, act as bioactive compounds that interact with biological aspects. These interactions in turn provide protective properties against cardiovascular disease [8]. There has even been investigation into the employment of functional foods in treating, preventing, and managing cancer. Many herbal remedies such as leaves and bark are considered functional foods. Cancer patients have been using these herbal remedies to manage cancers, including breast, colon, and prostate cancer. The functional food remedies have been clinically shown to help these patients in management, solidifying the importance of functional foods in managing chronic disease [9]. The bioactive compounds are the primary source of functional foods being able to treat, manage, and prevent chronic disease. Consequently, bioactive compounds must be acknowledged to fully understand the concept of functional foods [1].

Bioactive compounds interact with biomarkers. In doing this, these compounds can further enhance people's quality of life, which is exhibited in clinical research. Bioactive compounds are the driving force that establishes the functional aspect of functional foods. A standard acknowledgement of these compounds will bring a general understanding as to how functional foods improve health. Thus, the Functional Food Center's updated definition is a stride in the right direction. Bioactive compounds have an essential role in functional foods as the component that improves health and helps to treat disease [1-2].

\section{REFERENCES}

1. Danik Martirosyan and Kasia Pisarski: Bioactive Compounds: Their Role in Functional Food and Human Health, Classifications, and Definitions. In: Bioactive Compounds and Cancer. Edited by Danik Martirosyan and Jin-Rong Zhou. San Diego: Food Science Publisher; 238-277.

2. Danik Martirosyan and Jaishree Singh: A new definition of functional food by FFD: what makes a new definition unique? Functional Foods in Health and Disease 2015; 5(6):209-223

3. J. A. Milner: Functional Foods and Health Promotion, The Journal of Nutrition, Volume 129, Issue 7, 1 July 1999, Pages 1395S-1397S.

4. Khan, M.I., Anjum, F.M., Sohaib, M. et al.: Rev Endocr Metab Disord (2013) 14: 287.

5. Institute of Food Technologists. Functional foods: Opportunities and challenges. March 2005. 
6. Del Castillo MD, Iriondo-DeHond A, Martirosyan DM: Are Functional Foods Essential for Sustainable Health?. Ann Nutr Food Sci. 2018; 2(1): 1015.

7. Alkhatib, A., Tsang, C., Tiss, A., Bahorun, T., Arefanian, H., Barake, R., et al.: Functional Foods and Lifestyle Approaches for Diabetes Prevention and Management. Nutrients, 2017 9(12), 1310.

8. Cardoso, S.M.; Pereira, O.R.; Seca, A.M.L.; Pinto, D.C.G.A.; Silva, A.M.S: Seaweeds as Preventive Agents for Cardiovascular Diseases: From Nutrients to Functional Foods. Mar. Drugs 2015, 13, 6838-6865.

9. Clement, Y. N., Mahase, V., Jagroop, A., Kissoon, K., Maharaj, A., Mathura, P., et al.: Herbal remedies and functional foods used by cancer patients attending specialty oncology clinics in Trinidad. BMC Complementary and Alternative Medicine, 2016 16, 399. 